\title{
Uma Nova Antena Fractal para Aplicação em Televisão Digital Móvel na Banda VHF
}

\author{
Manoel J.L. Alves, Késia C. S. Farias, Carlos D. M. Regis, Marcelo S. de Alencar, Marcelo B. Perotoni \\ Instituto de Estudos Avançados em Comunicações, Universidade Federal de Campina Grande, \\ Av. Aprígio Veloso, 882, Campina Grande - PB
}

\begin{abstract}
Resumo-Este trabalho apresenta o projeto de uma nova antena fractal retangular (AFR) para televisão (TV) digital móvel na banda VHF, para uso em dispositivos móveis. As antenas para essas aplicações devem possuir dimensões pequenas e perfil plano. Resultados do diagrama de radiação nos planos $\mathbf{E}$ e $\mathbf{H}$ e do ganho (dB) na banda VHF são apresentados de forma a validar a aplicação dessa estrutura em TV digital móvel.
\end{abstract}

Palavras-Chave-Antena fractal, televisão móvel, banda VHF, Planos E e H.

Abstract-This paper presents the design of a new rectangular fractal antenna for digital television (TV) in VHF band to applications on mobile devices. The antennas for these applications must have small dimensions and low profile to be coupled with such devices. Results of the diagram of radiation on the $H$ and $E$ plans and gain (dB) of VHF band are presented in order to validate the application of this structure in mobile digital TV.

Keywords - Fractal antenna, mobile television, VHF band, $\mathrm{H}$ and $\mathbf{E}$ planes.

\section{INTRODUÇãO}

As vantagens da antena de microfitas tais como: volume pequeno, peso reduzido, baixo custo, facilidade de fabricação, configuração planar e compatibilidade com circuitos integrados têm feito dela muito popular e atraido a pesquisa científica por vários anos [1]. Embora com os meritos acima mencionados seria esperado imaginar essa antena como uma boa candidata para muitas aplicações, mas sua grande dimensão física torna-a imprópria quando a disponibilidade de espaço é uma limitação.

Por isso, vários métodos têm sido considerados para reduzir o tamanho da antena, como a utilização de pinos anti-curtos (shorting pins) e otimização geométrica [1]-[2]. Ainda que, seja interessante fazer menção do esforço de aumentar o percurso condutor da antena que foi tentado por meio da introdução de fendas e chanfras. Foi recentemente, com a introdução de fractais na engenharia, de antenas, que isso pode ser feito de uma maneira mais eficiente [3]. As formas fractais se revelaram ter maior dimensionalidade do que a euclidiana, em outras palavras elas podem explorar de forma mais eficiente uma área finita ou volume.

A análise de antenas para utilização em dispositivos móveis para recepção de televisão (TV) digital deve considerar características de operação na banda de frequiência desejada VHF, UHF e na banda de celular ( $800 \mathrm{MHz}$ e $1.8 \mathrm{GHz}$ ), mantendo

M. J. L. Alves, jacinto@ iecom.org.br, Tel +55-83-3310-1579, K. C. Santos, C. D. M. Regis, M. S. Alencar, malencar@iecom.org.br, Tel +55-83-33101410; Este trabalho foi financiado pela CAPES, CNPq, IESAM e Iecom. as dimensões e pesos aceitáveis para uso nesses dispositivos, além de considerar a forma do diagrama de radiação e ganho.

A dificuldade principal no desenvolvimento de antenas para a recepção móvel de TV digital é obter uma antena compacta que trabalhe na faixa de televisão e de comunicações móveis e possua dimensões compatíveis com a dos aparelhos portáteis. Assim, as antenas fractais e compactas de microfita se mostram muito atraentes para essas aplicações [4].

A resposta das antenas fractais difere acentuadamente da resposta das tradicionais, pois são capazes de funcionar simultâneamente em várias frequências, além de poderem ser miniaturizadas mantendo os parâmetros físicos desejados [5]. Assim, nesse trabalho foi desenvolvida uma nova antena fractal para a utilização no sistema brasileiro de televisão digital (ISDTV) para dispositivos móveis, tanto telefones celulares quanto PDA's na banda de VHF.

Este trabalho apresenta uma breve descrição dos aspectos de TV digital móvel na seção II. A seção III faz uma análise da nova antena fractal para dispositivos móveis. Os resultados preliminares relativos ao comportamento elétrico da antena são apresentados na seção IV. A seção V apresenta as conclusões desta etapa de pesquisa.

\section{Televisão Digital Móvel}

A capacidade de multimídias em dispositivos móveis cresceu para incluir dados, áudio, vídeo e, agora, em tempo real, a programação de TV digital [6]. O sistema de TV digital móvel permite a transmissão de programas televisivos ou vídeo para dispositivos móveis, como telefones celulares e PDA's. Os programas podem ser transmitidos para cada espectador em uma área, na forma de download ou por radiodifusão. A transmissão pode ser feita por meio terrestre, como a TV analógica ou digital é transmitida, por intermédio de satélites, pela Internet ou pelas redes celulares.

Existem dois tipos de serviços que utilizam o termo TV móvel. O primeiro serviço usa download, em que os vídeos visualizados por meio de galerias que incluem uma miniatura do vídeo e um título descrevendo o conteúdo. Esse serviço utiliza redes celulares.

Para esse serviço é necessária a introdução de um guia, que auxilia o usuário na escolha do conteúdo desejado. A escolha do programa no guia pode ser um inibidor do consumo do serviço de TV móvel devido à visualização do conteúdo no guia ser feita por meio de uma imagem e de um título.

O segundo tipo de serviço baseia-se na transmissão terrestre, na qual os sinais são transmitidos em canais paralelos. A 
mudança para outro canal pode ser feita tanto por um atalho para o número do canal ou usando o botão para cima e para baixo do receptor. Se não houver cobertura em uma área não se pode usar esse serviço de TV móvel.

Os requisitos da transmissão de TV móvel são [7]:

- transmissão em formatos adequados para dispositivos móveis, como por exemplo, formatos QCIF, CIF ou QVGA;

- baixo consumo de energia;

- recepção estável com mobilidade, para velocidades de até $250 \mathrm{~km} / \mathrm{h}$ ou superiores;

- imagem com boa qualidade, mesmo quando houver perda do sinal devido ao efeito de múltiplos percursos;

- capacidade para receber o sinal em áreas distantes das antenas.

A necessidade de os receptores de TV móvel receberem sinais tanto da rede celular quanto da rede de TV digital faz com que os receptores tenham antenas com alto ganho e grande largura de faixa na recepção, para que a TV móvel possa ter a qualidade requerida pelos usuários. Um outro ponto que deve ser levado em conta são as dimensões dessas antenas, que devem ser proporcionais às dimensões do aparelho portátil.

Essas antenas devem combrir as bandas de frequência de TV digital (UHF e VHF) para o serviço de TV móvel e da rede celular $(1800 \mathrm{MHz})$. O padrão de TV digital adotado pelo Brasil é ISDTV, que é uma modificação do ISDB [8]. Para esse padrão é adotada uma banda de freqüência de $6 \mathrm{MHz}$ para cada canal sendo este dividido em 13 segmentos, o segmento central é destinado para a transmissão da TV móvel e os outros segmentos para a transmissão da TV digital. Esse esquema pode ser visto na Fig. 1. Por usar apenas um dos segmentos a TV móvel usado no ISDB é chamado de One Seg e cada segmento tem uma banda de $433 \mathrm{kHz}$.

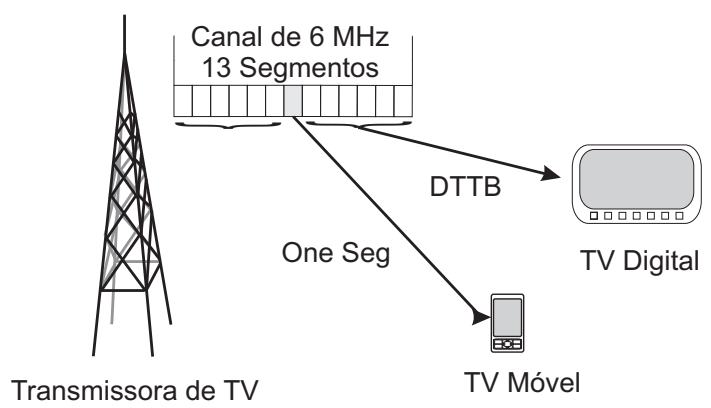

Fig. 1. Serviço ISDB-T.

Para a diminuição da antena pode-se usar antenas fractais, que possuem uma boa capacidade de miniaturização, sem reduzir significativamente a largura de banda e a eficiência da antena. A geometria dessa antena possui alguns graus de liberdade, proporcionando uma melhora em relação às antenas convencionais.

\section{ANTENA FRACTAL}

As técnicas de fractais são amplamente conhecidas na comunidade científica matemática. Entretanto, atualmente esse conceito tem sido usado para o projeto e a concepção de antenas. Essa técnica produz dois efeitos marcantes: reduz a dimensão física das estruturas radiantes e pode fazê-las funcionar em diferentes faixas de freqüências [9].

Recentemente, o projeto de antenas se beneficiou do estudo dessas geometrias, o que possibilitou a descoberta de antenas e de elementos bem mais eficientes do que os existentes. Além disso, os novos projetos permitem a redução da dimensão das antenas e facilitam o casamento de impedância. Certas classes de antenas fractais podem ser configuradas para efetivamente operar em diferentes faixas de freqüência [10].

Atualmente um dos principais desafios tecnológicos na construção de equipamentos eletrônicos para uso pessoal prende-se ao desenvolvimento de dispositivos móveis cada vez mais rápidos, menores e com maior número de serviços possíveis. Além disso, devido à atual exigência de mobilidade do usuário, os equipamentos sem fio são essenciais. Esse último requisito leva à necessidade de incorporar antenas que, simultaneamente, suportem todos os serviços desejados, e possam ser integradas no espaço disponível.

No entanto, o fato da dimensão ótima de uma antena ser diretamente proporcional ao comprimento de onda de trabalho é bem conhecido e portanto, para se obter o mesmo desempenho, quanto maior é a freqüência menor a dimensão da antena. Dessa forma, devido à crescente miniaturização dos sistemas eletrônicos de grande consumo, por exemplo telefones celulares e PDA's, o espaço disponível para as antenas é cada vez menor obrigando, muita vezes, à utilização de antenas de dimensões muito inferiores às ideais. Uma boa caracterização das antenas fractais, de modo a otimizar o seu desempenho face ao espaço disponível, é importante.

As antenas fractais impressas apresentam características que as tornam adequadas para integração em telefones celulares. O seu baixo perfil e a grande versatilidade no comportamento elétrico (largura de banda, diagrama de radiação, ganho, diretividade) tornou o uso desse tipo de antena muito útil em muitas aplicações comerciais.

\section{A. Antena de Microfita Retangular Fractal}

A geometria fractal envolve uma metodologia geradora recursiva que resulta em contornos com estruturas delgadas infinitamente intrincada. Essa geometria, que tem sido utilizada para modelar objetos complexos encontrados na natureza, tais como nuvens e orla costeira, tem propridades de preenchimento do espaço (space-filling), que podem ser utizadas para miniaturizar antenas [11].

Neste trabalho, uma nova antena fractal retangular (AFR) é analisada e examinada como uma candidata antena de microfita para aplicação em TV digital móvel na banda de VHF. A configuração proposta ilustrada na Fig. 2 foi construída aplicando uma transformação geométrica e redução do tamanho por um fator de escala, a partir de um iniciador retangular de área $220 \mathrm{~mm}^{2}(b \times c)$. Essa configuração foi obtida após três iterações fractais e, em cada iteração foi reduzida de tamanho por um fator de escala dado por

$$
S_{n}=\frac{D_{\circ}}{D_{n}},
$$


em que

$$
D_{\circ}=\frac{1}{2} \sqrt{b_{\circ}^{2}+c_{\circ}^{2}}
$$

E $b_{\circ}$ e $c_{\circ}$ a largura e comprimento, respectivamente, da antena de microfita fractal.

$\mathrm{E}$

$$
D_{n}=D_{\circ} \sum_{i=0}^{n} \frac{1}{2^{i}},
$$

em que $D_{n}$ é a semi-diagonal da $n$-ésima iteração.

Então,

$$
S_{n}=\left(\sum_{i=0}^{n} \frac{1}{2^{i}}\right)^{-1}
$$

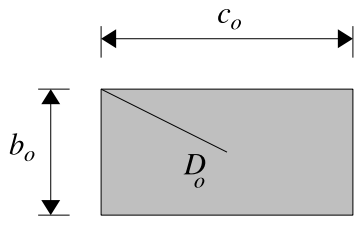

a)

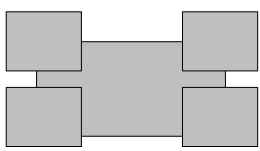

b)

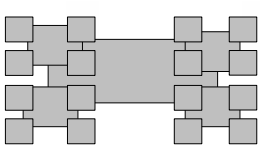

c)

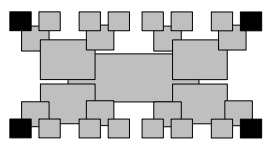

d)
Fig. 2. Três iterações fractais, a) iniciador $11 \mathrm{~mm} \times 20 \mathrm{~mm}$, b) primeira iteração (AFR-1), c) segunda iteração (AFR-2), d) terceira iteração (AFR-3), em cada iteração a forma é reduzida de tamanho por um fator de escala de $S_{\circ}=D_{\circ} / D_{n}$, em que $D_{n}$ é semi-diagonal da n-ésima iteração.

\section{Resultados das Simulações}

A AFR ilustrada na Fig. 3, consiste de um patch colocado uma fração pequena de comprimento de onda acima do plano terra. O patch e o plano terra são separados por um substrato dielétrico. O patch condutor é de cobre e sua forma é quadrada, enquanto o substrato é o FR-4 de permissividade elétrica relativa $\left(\varepsilon_{r}\right)$ igual a 4,9 . A geometria da antena é caracterizada por seu comprimento $c=20 \mathrm{~mm}$, largura $b=11 \mathrm{~mm} \mathrm{e}$ espessura $v=1,3146 \mathrm{~mm}$. Essa antena é formada por quatro elementos irradiantes quadrados de lado $a=2 \mathrm{~mm}$ sobre o substrato. Ela foi simulada na banda de VHF $(54-216 \mathrm{MHz})$ na freqüência de operação de $190 \mathrm{MHz}$.

Os resultados do diagrama de radiação da diretividade do plano-E e do plano-H são apresentados nas Figs. 4 e 5, respectivamente. Dos resultados verifica-se que a AFR possui um comportamento omnidirecional nos dois planos. Isso vem atender à espectativa quanto a resposta dessa antena, visto que, nesse estágio a antena exibiu uma redução do tamanho de $41,3 \%$ na terceira iteração. Nesse tipo de investigação qualquer mudança na posição e na dimensão dos irradiantes modifica sua resposta, ou seja, muda a freqüência de ressonância e, conseqüêntemente o comportamento elétrico da antena. Na Fig. 6 é ilustrado o ganho (dB) versus as
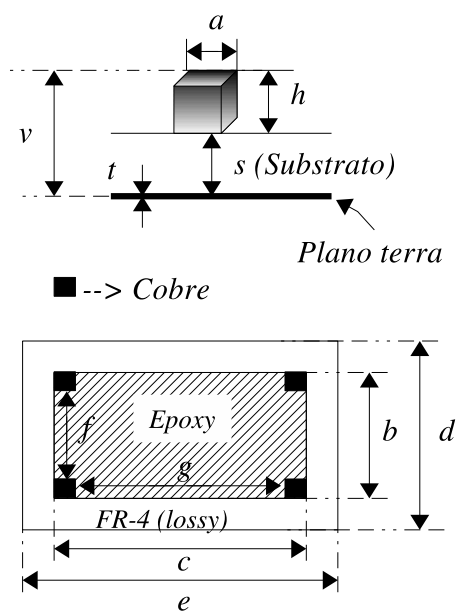

Fig. 3. Geometria de uma nova antena fractal: dimensões físicas da antena $a=2 \mathrm{~mm}, b=11 \mathrm{~mm}, c=20 \mathrm{~mm}, d=16 \mathrm{~mm}, e=22 \mathrm{~mm}, f=6 \mathrm{~mm}$, $g=15 \mathrm{~mm}, h=1 \mathrm{~mm}, t=0,0286 \mathrm{~mm}, s=0,286$ e $v=1,3146 \mathrm{~mm}$.

freqüências $(\mathrm{MHz})$ da AFR na banda VHF do padrão brasileiro de TV para uso em dispositivos móveis. A AFR possui uma largura de banda passante de aproximadamente $360 \mathrm{MHz}$.

O software utilizado para simulação da AFR foi o CST MICROWAVE STUDIO, com solver freqüência/tempo. O tempo de simulação foi 102,94 nanosegundos em um computador AMD Semptron (tm) Processor 3000+, 1,80 GHz, $1 \mathrm{~GB}$ de RAM.

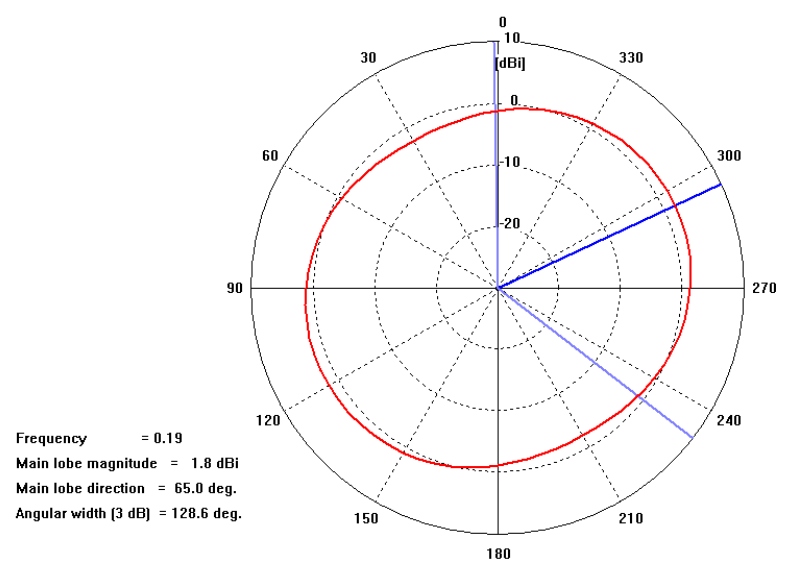

Fig. 4. Diagrama de radiação do plano-E de uma antena fractal com frequêencia de ressonância igual a $190 \mathrm{MHz}$ na banda VHF.

\section{CONClusões}

A etapa principal da investigação é o desenvolvimento de uma AFR para utilização em dispositivos móveis, operando nas frequiências da banda VHF. Foram realizadas simulações para essa antena, em que se verificou as respostas de sua diretividade nos planos $\mathrm{E}$ e $\mathrm{H}$ e do ganho como satisfatórias para a fase de implementação. A resposta da AFR é apresentada por meio do seu comportamento omnidirecional nos dois planos. 


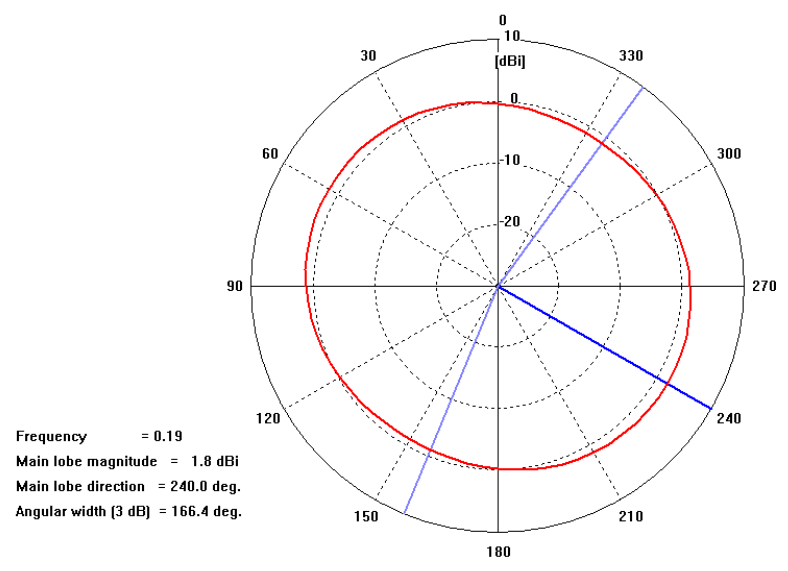

Fig. 5. Diagrama de radiação do plano-H de uma antena fractal com frequiência de ressonância igual a $190 \mathrm{MHz}$ na banda VHF.

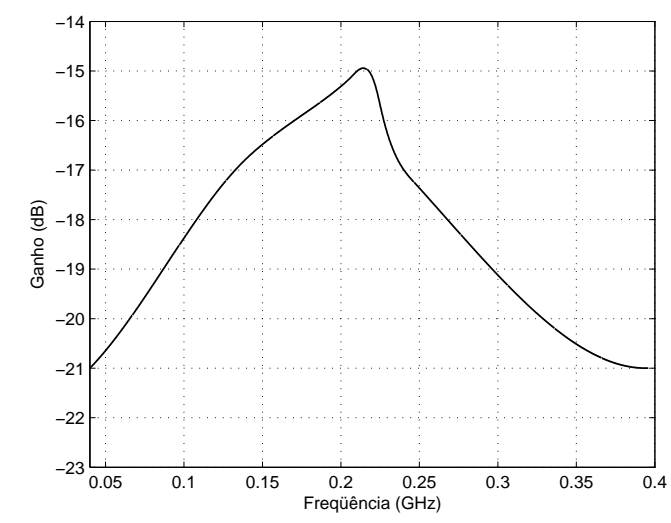

Fig. 6. Ganho (dB) da antena fractal retangular na banda VHF.

Além disso, a nova geometria tem diversos graus de liberdade que podem ser usados para reduzir mais a dimensão da antena ou ajustar a largura de banda.

\section{AGRADECIMENTOS}

Os autores agradecem o apoio do Conselho Nacional de Desenvolvimento e Pesquisa (CNPq), do Instituto de Estudos Superiores da Amazônia (IESAM) e do Instituto de Estudos Avançados em Comunicações (Iecom).

\section{REFERÊNCIAS}

[1] I.J. Bahl e P. Barthia, "Microstrip Antennas", Artech House, 1982.

[2] A. K. Skrivervik et al, "PCS Antenna Design: The Challenge of Miniaturization", IEEE Antennas and Propagation Magazine, 43(4):1227, August 2001.

[3] J. P. Gianvittorio, R-S. Yahya, "Fractal Antennas: A Novel Antenna Miniaturization Technique, and Applications", IEEE Antennas and Propagation Magazine, 44(1):20-35, February 2002.

[4] K. L. Wong, "Compact and Broadband Microstrip Antennas", John Wiley \& Sons, 2002.

[5] G. Tsachtsiris, C. Soras, M. Karaboikis and V. Makios, A Reduced Size Fractal Rectangular Curve Patch Antenna, IEEE, 2003.

[6] S-G. Jeon et al, "Broadband Internal Antenna for Mobile DTV Handsets", Progress In Electromagnetics Research Symposium (PIERS), 3(7):1048-1052, March 2007.

[7] Amitabh Kumar: "Mobile TV: DVB-H, DMB, 3G Systems and Rich Media Applications", Focal Press Media tecnology Professional, 2007.
[8] M. S. Alencar, ”Televisão Digital”. São Paulo: Editora Érica, 2007.

[9] L. Josefsson, P. Persson, Conformal Array Antenna Theory and Design, John Wiley \& Sons, Inc., Hoboken, New Jersey, 2006.

[10] C. Puente-Baliarda et al, "On the behavior of the sierpinski multiband fractal antenna", IEEE Transactions on Antennas and Propagation, 46(4):517-524, April 1998.

[11] D. H. Werner, R. L. Haupt, P. L. Werner, "Fractal Antenna Engineering: The Theory and Design of Fractal Antenna Arrays", IEEE Antenas and Propagatioon Magazine, 41(5):37-59, Octuber 1999. 